\title{
Increased expression of ATP12A proton pump in cystic fibrosis airways
}

\author{
Paolo Scudieri, ${ }^{1}$ Ilaria Musante, ${ }^{1}$ Emanuela Caci, ${ }^{2}$ Arianna Venturini, ${ }^{1}$ Patrizia Morelli, ${ }^{3}$ \\ Christine Walter, ${ }^{4,5}$ Davide Tosi, ${ }^{6}$ Alessandro Palleschi, ${ }^{6}$ Pablo Martin-Vasallo, ${ }^{7}$ \\ Isabelle Sermet-Gaudelus, ${ }^{8}$ Gabrielle Planelles, ${ }^{4,5}$ Gilles Crambert, ${ }^{4,5}$ and Luis J.V. Galietta ${ }^{1}$ \\ ${ }^{1} T e l e t h o n$ Institute of Genetics and Medicine (TIGEM), Pozzuoli NA, Italy. ${ }^{2}$ U.O.C. Genetica Medica, Istituto Giannina Gaslini, \\ Genova, Italy. ${ }^{3}$ U.O.C. Laboratorio Analisi, Istituto Giannina Gaslini, Genova, Italy. ${ }^{4}$ CNRS ERL 8228 - Centre de Recherche \\ des Cordeliers - Laboratoire de Métabolisme et Physiologie Rénale, Paris, France. ${ }^{5}$ Sorbonne Université, UPMC Univ Paris \\ 06, INSERM, Université Paris Descartes, Sorbonne Paris Cité, UMR_S 1138, Centre de Recherche des Cordeliers, Paris, France. \\ ${ }^{6}$ Thoracic Surgery and Lung Transplantation Unit, Fondazione IRCCS Ca' Granda - Ospedale Maggiore Policlinico, Milano, \\ Italy. ${ }^{7}$ UD de Bioquímica y Biología Molecular and Centro de Investigaciones Biomédicas de Canarias (CIBICAN), Universidad \\ de La Laguna, La Laguna, Tenerife, Spain. ${ }^{8}$ Institut Necker Enfants Malades/INSERM U1151, Paris, France.
}

Proton secretion mediated by ATP12A protein on the surface of the airway epithelium may contribute to cystic fibrosis (CF) lung disease by favoring bacterial infection and airway obstruction. We studied ATP12A in fresh bronchial samples and in cultured epithelial cells. In vivo, ATP12A expression was found almost exclusively at the apical side of nonciliated cells of airway epithelium and in submucosal glands, with much higher expression in CF samples. This could be due to bacterial infection and inflammation, since treating cultured cells with bacterial supernatants or with IL-4 (a cytokine that induces goblet cell hyperplasia) increased the expression of ATP12A in nonciliated cells. This observation was associated with upregulation and translocation of ATP1B1 protein from the basal to apical epithelial side, where it colocalizes with ATP12A. ATP12A function was evaluated by measuring the $\mathrm{pH}$ of the apical fluid in cultured epithelia. Under resting conditions, CF epithelia showed more acidic values. This abnormality was minimized by inhibiting ATP12A with ouabain. Following treatment with IL-4, ATP12A function was markedly increased, as indicated by strong acidification occurring under bicarbonate-free conditions. Our study reveals potentially novel aspects of ATP12A and remarks its importance as a possible therapeutic target in CF and other respiratory diseases.

Conflict of interest: The authors declare that there is no conflict of interest.

Submitted: July 16, 2018 Accepted: August 29, 2018 Published: October 18, 2018

\section{Reference information:} JCI Insight. 2018;3(20):e123616. https://doi.org/10.1172/jici. insight.123616.

\section{Introduction}

The airway surface is covered by a thin layer of liquid (airway surface liquid; ASL), whose ion composition plays an important function in the innate defense mechanisms against microbial agents (1). Ion composition of ASL is controlled by the coordinated activity of ion channels, transporters, and pumps. Among such membrane proteins, the function of the CFTR anion channel is particularly critical. In cystic fibrosis (CF), one of the most frequent genetic diseases, loss of CFTR-dependent $\mathrm{Cl}^{-}$secretion causes dehydration of airway surface and impairment of mucociliary clearance. CFTR is also involved in bicarbonate secretion, directly or through other transporters (e.g., SLC26A4) that exchange $\mathrm{Cl}^{-}$secreted by CFTR with intracellular bicarbonate (2-5). Defective bicarbonate secretion may cause ASL acidification with multiple negative consequences, including impairment of antimicrobial mechanisms and production of a dense, difficult to clear mucus (6-8). In contrast with various studies reporting an abnormality in ASL $\mathrm{pH}$, a recent paper has shown no difference in $\mathrm{pH}$ between $\mathrm{CF}$ and non-CF airways (9). The reason for such contrasting results is, at the moment, unclear.

ATP12A - the nongastric form of the $\mathrm{H}^{+} / \mathrm{K}^{+}$-ATPase (10), which is localized on the apical membrane of airway epithelial cells $(3,5,11)$ - is one of the ion transporters involved in ASL pH regulation. ATP12A is emerging as an important pathogenic factor in CF and other chronic respiratory diseases such as asthma $(3,12,13)$. Indeed, in normal airways, the proton secretion operated by ATP12A is expected to be compensated by the parallel bicarbonate transport. In CF patients, loss of CFTRdependent bicarbonate secretion leaves proton secretion unchecked. Interestingly, ATP12A was found to be expressed in human and pig, but not in murine, airways (3). The absence of ATP12A in mice could explain the lack of a human-like lung disease in animals devoid of CFTR function. 
We previously found that ATP12A expression in cultured bronchial epithelial cells is strongly upregulated by IL-4, particularly in nonciliated mucus-producing cells (3). More recently, this type of response was also found for IL-13 (13). IL-4 and IL-13, 2 cytokines involved in Th2 immune response and the development of bronchial asthma, are known to induce goblet cell hyperplasia $(14,15)$, a process that also involves upregulation of many other ion channels and transporters (e.g., TMEM16A/ANO1, SLC26A4/ pendrin, SLC12A2) associated with $\mathrm{Cl}^{-}$and bicarbonate secretion $(5,16)$. It is believed that bicarbonate secretion is important in the release and expansion of mucins $(5,7,17,18)$. In a recent in vitro study, strong ATP12A induction by IL-13 was found to be associated with increased mucus viscosity (13). Therefore, because of usually elevated IL-13 level in asthmatic patients, it can be hypothesized that an abnormally high ATP12A function in vivo contributes to airway obstruction in asthmatic patients. Furthermore, the acidification mediated by ATP12A in CF ASL could directly predispose to mucus hyperviscosity and bacterial infections by impairing antibacterial activity of specialized peptides (3).

We have carried out studies on respiratory cultured cells and ex vivo samples to unravel various aspects related to ATP12A expression and function. Our findings demonstrate that ATP12A expression is increased in the airways of CF patients as a consequence of bacterial infection and inflammation. Furthermore, our studies reveal that ATP12A expression in the plasma membrane requires the association with ATP1B1, a $\beta$ subunit of $\mathrm{Na}^{+} / \mathrm{K}^{+}$-ATPase $(19,20)$. We also find a more acidic $\mathrm{pH}$ in the apical fluid of $\mathrm{CF}$ epithelia in vitro, an abnormality that can be contrasted by pharmacological inhibition of ATP12A. Finally, we found that ATP12A upregulation is associated with depletion of $\mathrm{K}^{+}$in ASL, an effect that, in addition to proton pumping, may have an important role in the barrier function of airway epithelium.

\section{Results}

We investigated the expression of ATP12A in fresh bronchial samples obtained from $\mathrm{CF}$ and non-CF individuals undergoing lung transplant. After lung resection, a piece of bronchial wall was fixed and processed for immunodetection of ATP12A and other epithelial proteins, particularly acetylated tubulin and MUC5AC, to identify ciliated and goblet cells, respectively. We collected samples from $15 \mathrm{CF}$ patients and from 8 individuals transplanted for other diseases, including idiopathic fibrosis, pulmonary sarcoidosis, and scleroderma. Figure 1 shows representative images of the bronchial surface and submucosal glands from multiple CF and non-CF bronchi. Quantification of ATP12A expression is reported in Figure 2A. ATP12A immunostaining was clearly detectable in the surface epithelium of CF patients (Figure 1). ATP12A was present almost exclusively in nonciliated cells, frequently associated with MUC5AC (Figure 2B). In contrast, ATP12A signal was hardly detectable in the surface epithelium of non-CF individuals (Figures 1 and Figure 2A). A notable exception is represented by patient HBE4, whose epithelium showed a clear apical membrane staining (Figure 1). To explain this finding, we inspected the clinical information available for this individual. Importantly, we found that HBE4 was affected by IgG deficiency and recurrent bacterial infections. This finding suggested that ATP12A hyperexpression can be directly induced in epithelial cells by bacteria and/or through a host response to infection involving cytokine release.

We also found evidence for ATP12A expression in the lumen of submucosal glands (Figures 1 and Figure 2C), a finding never reported before. In submucosal glands, the difference between $\mathrm{CF}$ and non-CF samples was less dramatic compared with surface epithelium. In particular, non-CF patients HBE4, HBE6, and HBE7 displayed an extent of ATP12A expression comparable with that of CF patients (Figures 1 and Figure 2C).

We investigated ATP12A expression in cultured bronchial epithelial cells from CF and non-CF individuals. By immunofluorescence, we found no evidence of increased ATP12A expression in CF cells kept under resting conditions (Figure $3 \mathrm{~A}$ ). To mimic bacterial infection, we treated cells with a bacterial supernatant and, for comparison, with IL-4. As shown before (5), IL-4 induced a strong increase in ATP12A expression, particularly in nonciliated cells. The bacterial supernatant also induced ATP12A expression, in both CF and non-CF cells, although less than IL-4. To confirm these results, ATP12A protein levels were also assessed by immunoblots. The results from multiple samples were in agreement with those obtained by immunofluorescence: treatment with the bacterial supernatant and, to a larger extent, with IL-4 similarly enhanced ATP12A expression in both CF and non-CF cells (Figure 3, B and C).

It is known that trafficking of ATP12A to the plasma membrane requires assembly with a $\beta$ subunit. However, identity of this subunit is controversial. Possible candidates are the $\beta$ subunit of the gastric $\mathrm{K}^{+} /$ $\mathrm{H}^{+}$-ATPase (i.e., ATP4B) or one of the $\beta$ subunits of the $\mathrm{Na}^{+} / \mathrm{K}^{+}$-ATPase, known as ATP1B1, ATP1B2, and ATP1B3 $(10,21-24)$. We reasoned that the correct partner of ATP12A in the airway epithelium also 


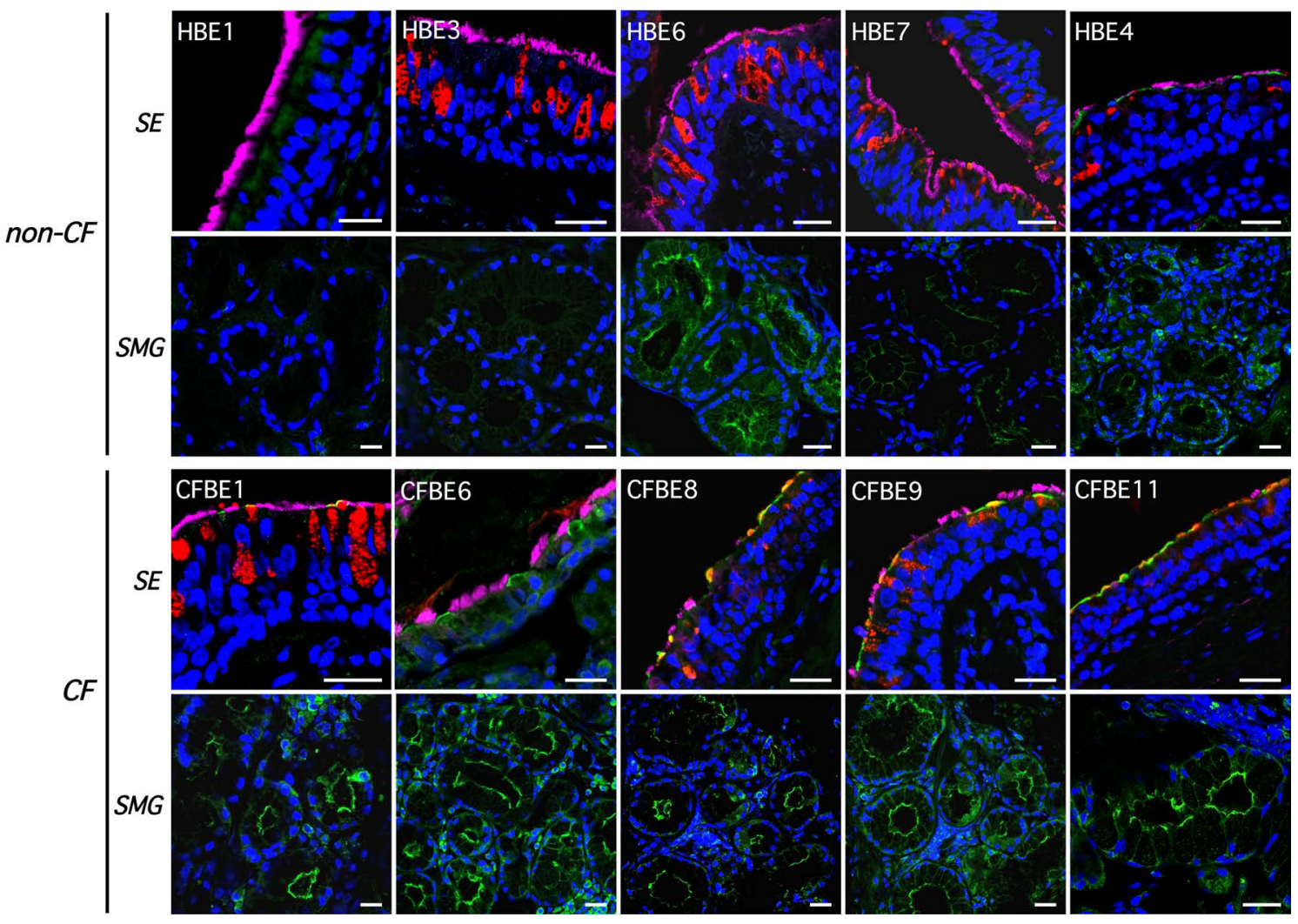

Figure 1. ATP12A protein expression in human bronchi. Representative confocal microscope images showing immunodetection of ATP12A (green), MUC5AC (red), and acetylated tubulin (magenta) in bronchial histological sections. Nuclei were counterstained with DAPI (blue). Scale bar: $20 \mu \mathrm{m}$. Images show the surface epithelium (SE) and submucosal glands (SMG) for different non-CF (top) and CF (bottom) bronchi. ATP12A expression was found in the apical membrane of all CF samples and of 1 non-CF bronchus (HBE4 individual). ATP12A was also expressed in the lumen of submucosal glands, particularly in those of CF patients.

had to be localized in the apical membrane and, possibly, upregulated by IL- 4 . We investigated the extent of expression and localization of $\beta$ subunits by immunofluorescence (Figure 4). Only 1 subunit, ATP1B1, appeared to be strongly upregulated by IL-4. Interestingly, ATP1B1 underwent a dramatic shift in subcellular localization. As evident from $x y$ and $x z$ confocal sections (Figure 4A), ATP1B1 was largely localized in the basolateral membrane of untreated cells. Treatment with IL-4 markedly increased the expression of ATP1B1 in the apical membrane. Figure 4B shows that this change is not caused by a general perturbation of cell polarity, since the $\mathrm{Na}^{+} / \mathrm{K}^{+}$-ATPase, ATP1A1, remained in the basolateral membrane of cells treated with the cytokine.

We further investigated the relationship between ATP12A and ATP1B1 by staining both proteins within the same cell preparation (Figure 5). In control cells, ATP1B1 was mostly present in the basolateral membrane. In a small fraction of cells, ATP1B1 was present in the apical membrane and colocalized with ATP12A. Upon treatment with IL-4, an increased number of cells showed a strong apical signal for both ATP12A and ATP1B1. To get further support for colocalization of the 2 proteins, we used superresolution microscopy by structured illumination microscopy (SIM) (Figure 5B). We took images from an apical plane, identified by staining of cilia, and from a more basal plane. Both ATP12A and ATP1B1 signals showed strong overlap in the apical plane, whereas only ATP1B1 was visible in the basal plane (Figure 5B). We also evaluated the ATP1B1 expression in freshly excised bronchial samples. In CF airways, ATP1B1 was clearly present in the basolateral membrane but was also coexpressed in the apical membrane, together with ATP12A (Figure 5C).

We also investigated ATP12A and ATP1B1 interaction using the proximity ligation assay proximity ligation assay (PLA) (Figure 6A). This method generates a fluorescent signal when 2 proteins, recognized by separate primary antibodies, are very close to each other. The proximity of the 2 antibodies allows a rolling circle DNA synthesis and subsequent binding of a fluorescent probe to amplified DNA. Using antibodies against ATP12A and ATP1B1, we clearly detected bright fluorescent spots, which indicate close 
A

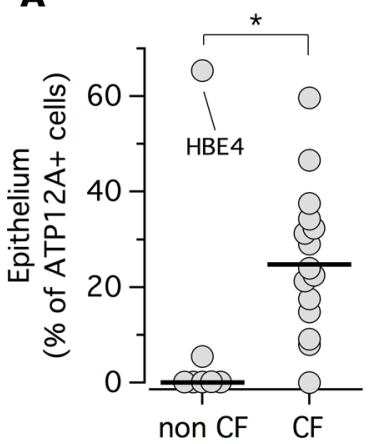

B

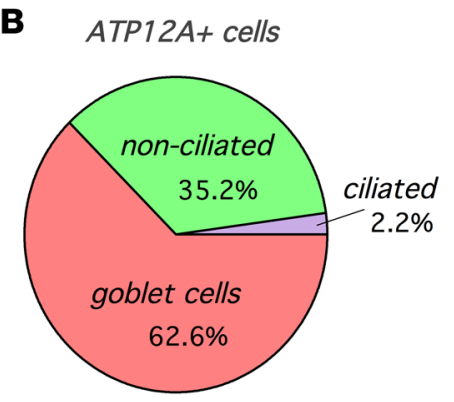

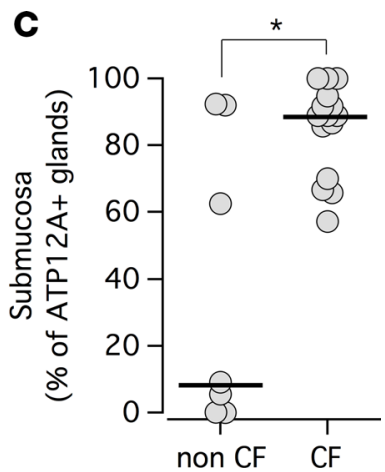

Figure 2. Quantification of ATP12A protein expression in human bronchi. (A) Dot plot reporting ATP12A expression (percent of ATP12A+ cells) in surface epithelia of non-CF and CF samples ( $n=8$ and 15, respectively); ${ }^{*}<0.05$ by nonparametric test. (B) Pie chart showing expression of ATP12A in specific cell types of the surface epithelium. (C) Dot plot reporting ATP12A expression (percent of ATP12A $A^{+}$glands) in submucosal glands of non-CF $(n=8)$ and CF $(n=15)$ patients; ${ }^{*} P<0.05$ by nonparametric test. Horizontal bars in dot plots indicate median values.

physical association between the 2 proteins. As expected, such spots were more numerous in cells treated with IL-4. Importantly, no spots were visible when the antibody for ATP4B was used in place of that for ATP1B1 (Figure 6A). A quantification of PLA data is shown in the dot plot of Figure 6A.

The results obtained in bronchial epithelial cells suggested a possible requirement of ATP1B1 for the trafficking of ATP12A. When ATP12A was transfected alone in bronchial CFBE41o- cells, we mostly found a perinuclear staining (Figure 6B, left). When both proteins were transfected together, we found appearance of ATP12A on the cell border, with a close overlap with ATP1B1 signal (Figure 6B, right).

The subcellular localization of ATP1B1 was also studied in the mouse colon (Figure 7). The $\beta$ subunit was localized in the apical membrane of WT mice, together with ATP12A. In contrast, the subunit showed an exclusive basolateral localization in ATP12A-KO mice.

To assess the function of ATP12A, we measured the $\mathrm{pH}$ of the apical fluid collected from cultured human bronchial epithelia kept under a $5 \% \mathrm{CO}_{2}$ atmosphere. With respect to a previous study (5), we kept the fluid on epithelia for only 3 hours (instead of 48 hours) and also included CF epithelia. The graph in Figure 8A compares the $\mathrm{pH}$ of $\mathrm{CF}$ vs. non- $\mathrm{CF}$ epithelia, with and without IL-4. We found that the $\mathrm{pH}$ of $\mathrm{CF}$ epithelia under unstimulated conditions was significantly more acidic than that of non-CF epithelia (median of 7.31 vs. 7.77). This finding is in agreement with previous studies, which reported an abnormal acidification in CF (11) but is different from a more recent paper showing no difference in $\mathrm{pH}(9)$. Intriguingly, cells stimulated with IL-4, both $\mathrm{CF}$ and non- $\mathrm{CF}$, showed a markedly alkaline fluid $(\mathrm{pH}>8)$ compared with untreated cells. Under these conditions, there was essentially no difference in $\mathrm{pH}$ between the 2 cell types (Figure 8A). The high $\mathrm{pH}$ value induced by IL-4 could be explained by an increased rate of bicarbonate secretion consequent to upregulation of several anion channels and transporters, including TMEM16A, SLC26A4, and SLC12A2 (5). To evaluate the net contribution of ATP12A to $\mathrm{pH}$, we carried out experiments without bicarbonate and also used apical ouabain as an inhibitor of the pump. The graphs in Figure 8, B and C, report the $\mathrm{pH}$ values for the different conditions. As expected, removal of bicarbonate caused acidification of the apical fluid. In untreated (no IL-4) cells, incubation with the bicarbonate-free basolateral solution decreased $\mathrm{pH}$ by nearly 0.5 $\mathrm{pH}$ units. Importantly, addition of apical ouabain blocked this acidification and brought the $\mathrm{pH}$ of $\mathrm{CF}$ and non-CF epithelia to similar values (Figure 8D). The $\mathrm{pH}$ change caused by bicarbonate removal was particularly dramatic for cells treated with IL-4, being equivalent to nearly a $1.4 \mathrm{pH}$ unit drop for both $\mathrm{CF}$ and non-CF epithelia. The absolute $\mathrm{pH}$ value under this condition (IL-4, no bicarbonate) was below 7 (median of 6.75 and 6.68 for non-CF and CF samples, respectively). This particular extent of acidification may be explained with the net activity of ATP12A (upregulated in cytokine-treated epithelia) not buffered by bicarbonate transport. In agreement with this assumption, apical ouabain significantly increased $\mathrm{pH}$ in the absence of bicarbonate. It should be noted that ouabain was ineffective in epithelia treated with IL-4 but kept with bicarbonate (Figure $8, \mathrm{~B}$ and $\mathrm{C}$ ), a surprising result since ATP12A was expected to be active under these conditions.

We also measured $\mathrm{K}^{+}$concentration in the apical fluid as another parameter reporting ATP12A activity (Figure 9). For this purpose, we used the same solution used for $\mathrm{pH}$ measurements, which contains $3 \mathrm{mM} \mathrm{K}^{+}$. After 3 hours on the apical side of untreated epithelia, the $\mathrm{K}^{+}$concentration of the fluid 
A

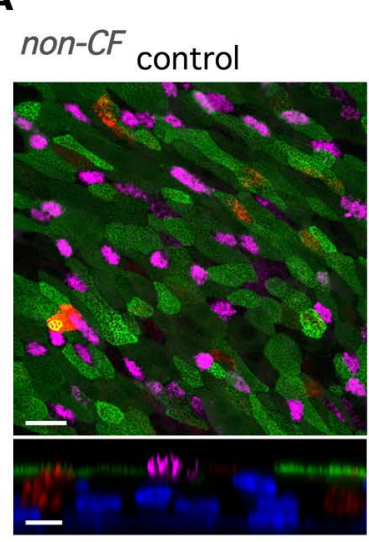

CF control

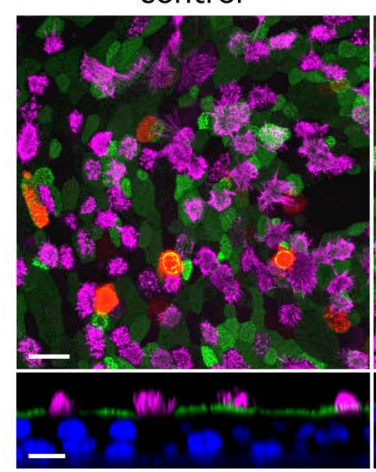

ATP12A MUC5AC cilia supernatant

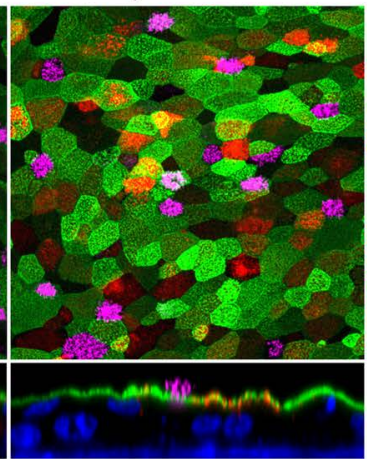

supernatant

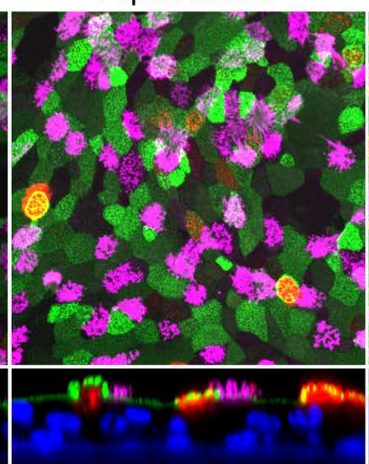

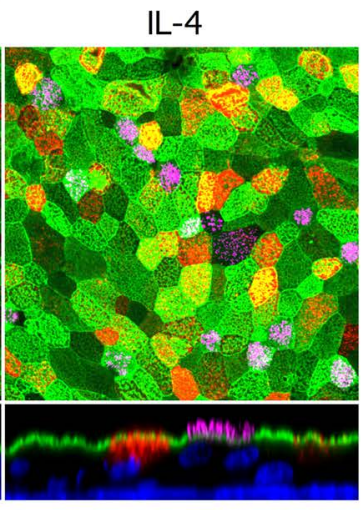

IL-4

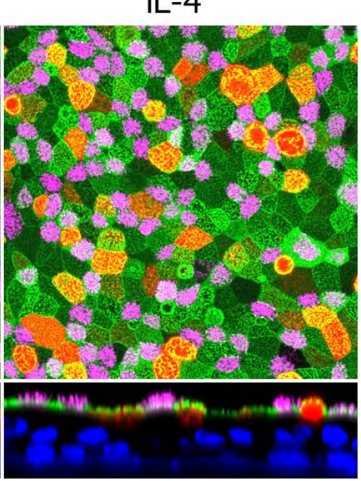

Figure 3. Expression of ATP12A protein in cultured bronchial epithelia. (A) Representative images showing detection of ATP12A (green), MUC5AC (red), and acetylated tubulin (magenta) by immunofluorescence. Images, taken with a confocal microscope, are $x y$ and $x z$ scans of non-CF (top) and CF (bottom) bronchial epithelial cells treated or not for 72 hours with bacterial supernatant or IL-4 $(10 \mathrm{ng} / \mathrm{ml})$. The $x z$ images also report staining of nuclei with DAPI (blue). Scale bar: $20 \mu \mathrm{m}$ and $10 \mu \mathrm{m}$ for $x y$ and $x z$ images, respectively. (B) Detection of ATP12A protein by Western blot in lysates from non-CF (left) and CF (right) bronchial epithelial cells under control condition or after 72 hours of treatment with bacterial supernatant (SN) or IL-4. GAPDH was also revealed as control. The full uncut images of these experiments are shown in the Supplemental Material (supplemental material available online with this article; https://doi.org/10.1172/jci. insight.123616DS1). (C) Dot plot showing the densitometric analysis of Western blot $(n=4$ for each condition). Data are normalized for GAPDH expression. ${ }^{*} P<0.05,{ }^{* *} P<0.001$ vs. control.

B

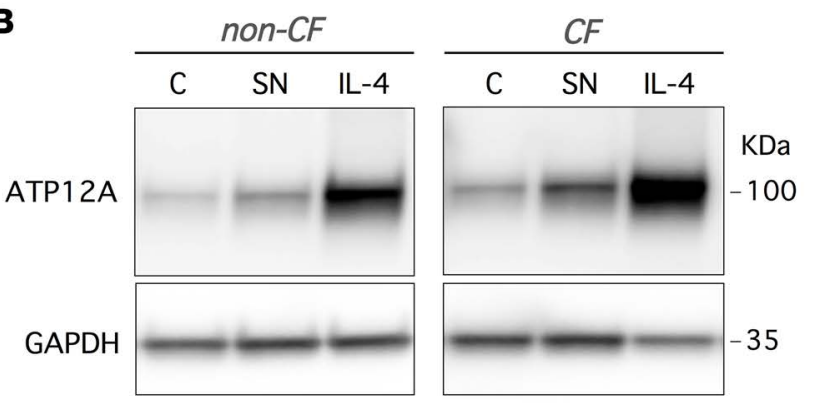

C $\bigcirc$ non-CF

- $C F$

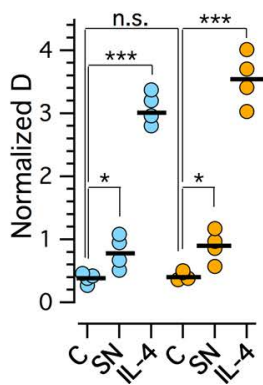

remained close to the initial value. Instead, the fluid of epithelia treated with IL-4 revealed a marked depletion of $\mathrm{K}^{+}$that, in many samples, was nearly undetectable (Figure 9). Importantly, this fall in $\mathrm{K}^{+}$ content was partially reduced by apical ouabain. In contrast to $\mathrm{K}^{+}, \mathrm{Na}^{+}$concentration was not significantly altered by IL-4 treatment (Figure 9).

\section{Discussion}

The epithelium covering the airways is an important defense barrier against potentially dangerous microbial agents delivered by inhaled air. In this respect, the physical and chemical properties of ASL (thickness, viscosity, small solutes, and macromolecules content) are finely controlled by the underlying epithelium and by submucosal glands. Throughout the years, the $\mathrm{pH}$ and bicarbonate concentration of ASL have increasingly emerged as important factors in CF lung disease $(3,6-8,17,18,25)$. A defect in bicarbonate secretion in CF airway epithelia was already described in 1992 (2). A low ASL pH value was later reported for $\mathrm{CF}$ epithelia (11). More recently, several studies have demonstrated that defective secretion of bicarbonate, due to CFTR lack of function, impairs innate defense mechanisms in multiple ways $(3,6,7)$. On the other hand, a recent study has shown no intrinsic difference in ASL pH between normal individuals and CF patients in vivo (9). 
A
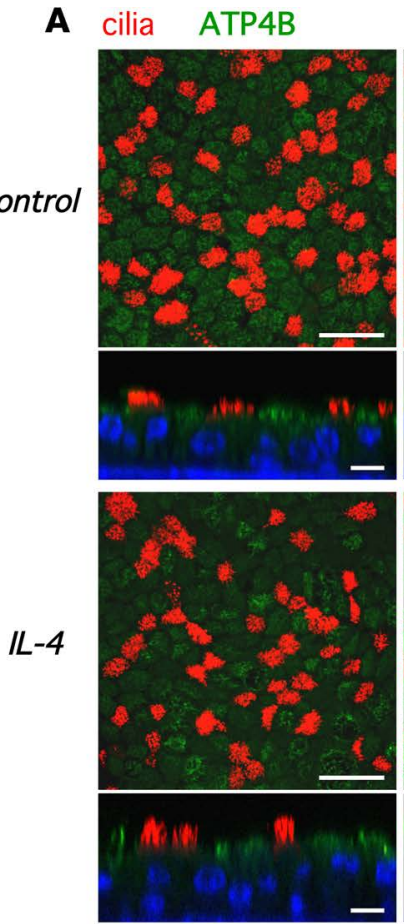

B

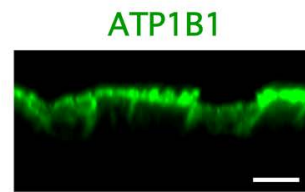

ATP1B1
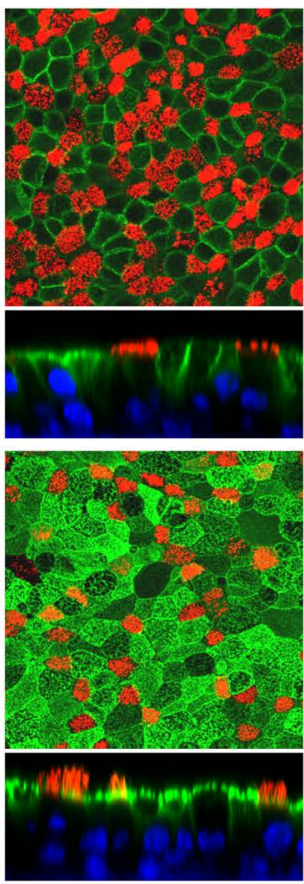

ATP1A1

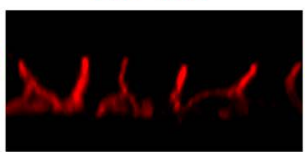

ATP1B2
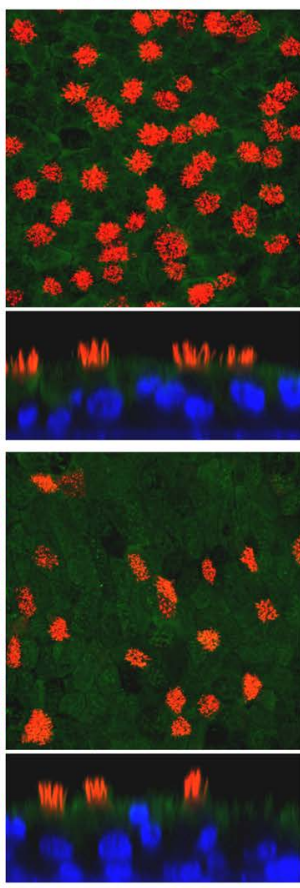

merge

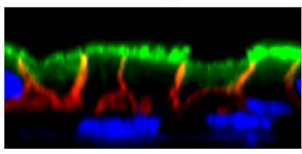

ATP1B3

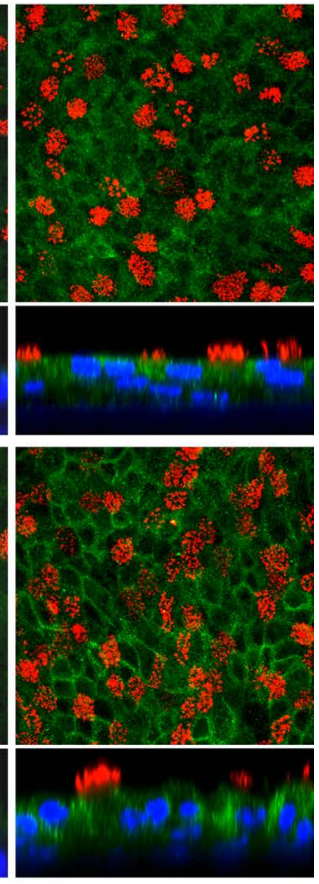

Figure 4. Expression of $\boldsymbol{\beta}$ subunits in cultured bronchial epithelial cells. (A) Representative $x y$ and $x z$ confocal images showing immunostaining for different candidate $\beta$ subunits (ATP4B, ATP1B1, ATP1B2, ATP1B3; green signal) and cilia (acetylated tubulin; red signal) in cultured bronchial epithelial cells treated with and without IL-4 for 72 hours. Scale bar: $40 \mu \mathrm{m}$ and $10 \mu \mathrm{m}$ for $x y$ and $x z$ images, respectively. IL-4 strongly increases the expression of the $\mathrm{Na}^{+} / \mathrm{K}^{+}-\mathrm{ATPase} \beta 1$ subunit (ATP1B1) in the apical membrane of epithelial cells. Data are representative of 3 separate preparations on nonCF cells and 2 preparations on CF cells. Images for ATP1B1 were obtained with the ab2873 antibody. Identical results were obtained with the SpETb1 antibody (not shown). (B) Immunofluorescence detection of $\mathrm{Na}^{+} / \mathrm{K}^{+}-\mathrm{ATPase} \alpha-$ and $\beta 1$ subunit (ATP1A1 and ATP1B1, respectively) in bronchial epithelial cells treated with IL-4. Scale bar: $10 \mu \mathrm{m}$.

In addition to CFTR, other channels and transporters are also involved in the secretion of bicarbonate and/or the control of ASL $\mathrm{pH}$. We recently investigated the gene expression changes and functional consequences elicited by IL-4, a cytokine that induces goblet cell hyperplasia and mucus hypersecretion (5). Our analysis revealed the increased expression and/or function of several membrane proteins involved in the transport of chloride and bicarbonate, including CFTR, TMEM16A, pendrin, and SCL12A2 (5, 16). Surprisingly, this analysis also evidenced the upregulation of ATP12A, which represents the nongastric form of the $\mathrm{K}^{+} / \mathrm{H}^{+}$-ATPase. Evidence for proton secretion coupled to $\mathrm{K}^{+}$absorption in the apical membrane of airway epithelial cells was previously reported in 2 studies $(11,26)$. More recently, it has been demonstrated that ATP12A protein is indeed responsible for proton secretion in human airways and, in the absence of CFTR-dependent bicarbonate transport, is a major cause of ASL acidification in CF $(3,27)$.

In the present study, we first investigated the expression of ATP12A in human airways. Interestingly, we found clear evidence of enhanced ATP12A levels in CF bronchi of patients with terminal disease and chronic infection, a characteristic that has not been reported before. In particular, ATP12A was found in nonciliated MUC5 $\mathrm{AC}^{+}$cells, in agreement with previous findings indicating its prevalent expression in goblet cells (5). The enhanced signal detected in CF airways could result from the combination of a higher number of goblet cells and an increased intrinsic expression within such cells. Enhanced ATP12A expression may not be directly associated to CFTR lack of function but rather seems a consequence of bacterial infection and, possibly, inflammation. Indeed, we saw no major differences in expression and function between $\mathrm{CF}$ and non-CF epithelia kept in vitro under sterile conditions. Instead, we observed induction of ATP12A expression when cells were exposed 
A

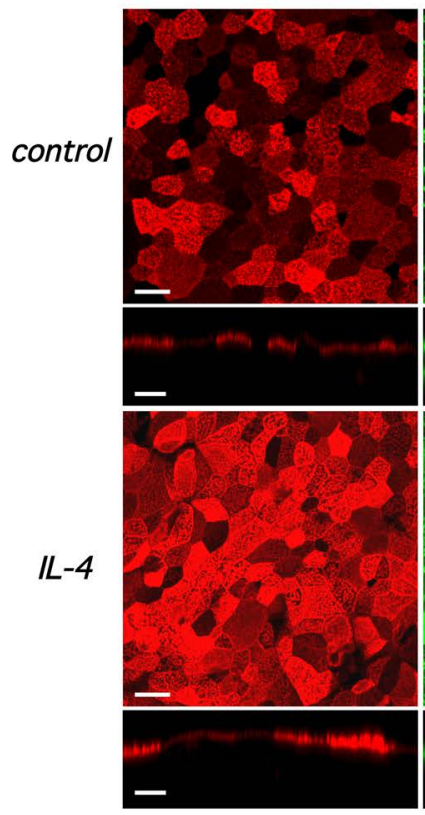

C

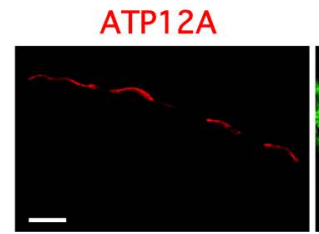

ATP1B1

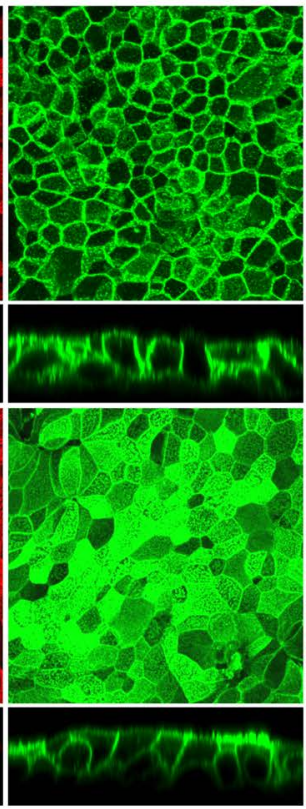

ATP1B1

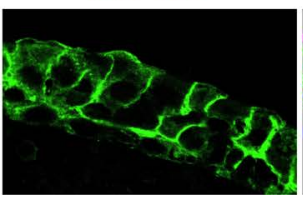

merge

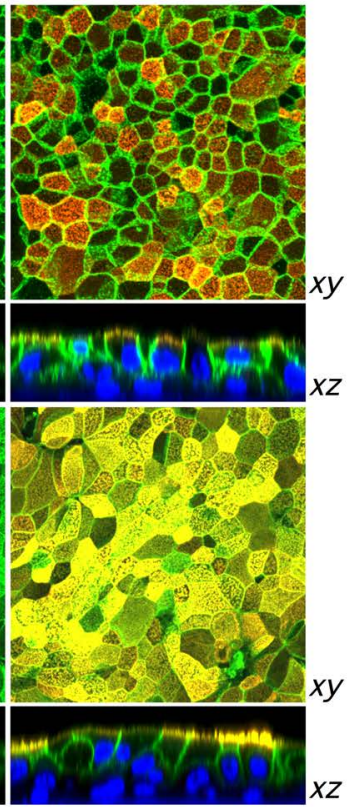

merge + cilia nuclei

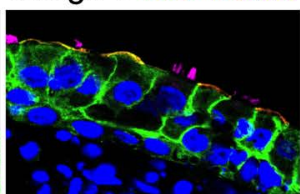

B ATP12A ATP1B1 cilia

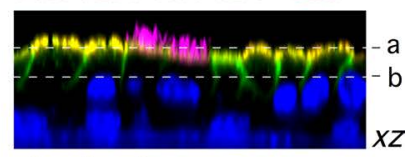

Confocal
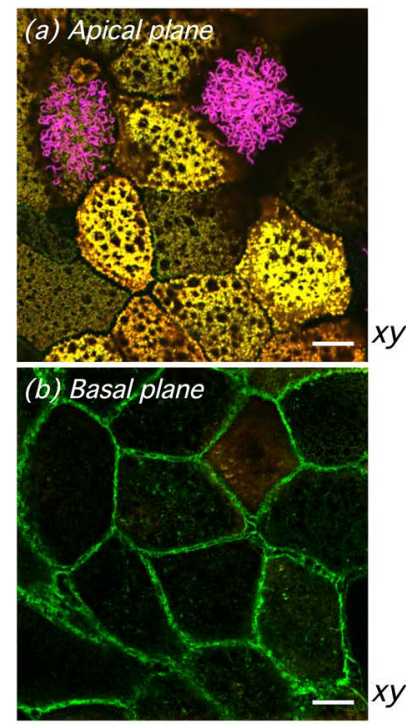

Superresolution

Figure 5. ATP12A-ATP1B1 colocalization in bronchial epithelial cells. (A) Representative $x y$ and $x z$ confocal images showing immunodetection of ATP12A and ATP1B1 in cultured bronchial epithelial cells under control condition (top) or after treatment with IL-4 for 72 hours (bottom). Scale bar: $20 \mu \mathrm{m}$ and 10 $\mu \mathrm{m}$ for $x y$ and $x z$ images, respectively. Images were collected from 3 separate preparations on non-CF cells and 2 preparations on CF cells. (B) Representative images taken by confocal $x z$ scans (top) and Elyra superresolution $x y$ scans (bottom) from cultured bronchial epithelial cells treated with IL-4. Images show colocalization of ATP12A and ATP1B1 in the apical membrane (apical plane) but not in the basolateral compartment (basal plane). Scale bar: $5 \mu$ m. (C) Representative images showing colocalization of ATP12A and ATP1B1 in CF bronchial samples. Scale bar: $10 \mu \mathrm{m}$.

to bacterial components. It is possible that ATP12A expression is similarly induced by bacterial components in vivo and further promoted by proinflammatory stimuli mimicking the effect of IL- 4 in vitro. Another interesting finding in our study of bronchial samples is the expression of ATP12A in the apical membrane of submucosal gland cells. This finding suggests that ATP12A also controls the composition and $\mathrm{pH}$ of gland secretions.

Our findings have an important implication. If ATP12A-mediated acidification reduces the antimicrobial activity on mucosal surface, as stated by Shah et al. $(3,27)$, then the upregulation that we have observed in ex vivo samples could imply a detrimental response that further worsens CF lung pathology. Therefore, we hypothesize that ATP12A should be a therapeutic target in CF and, more specifically, in chronically infected patients and during infection exacerbations.

A second aspect of ATP12A considered in our study was the identification of factors affecting its trafficking to the plasma membrane. Actually, there are different results regarding the identity of the subunit that is required by ATP12A to reach the cell surface. The different proteins proposed so far include ATP4B, ATP1B1, ATP1B2, and ATP1B3 (10, 21-24). Our results indicate that ATP1B1 is the partner of ATP12A. First, ATP1B1 was found to colocalize with ATP12A in the apical membrane of bronchial epithelial cells in vivo and in vitro, particularly in cultured cells treated with IL-4. Second, cotransfection with ATP1B1 is required to move ATP12A from intracellular compartments to the cell surface. ATP1B1 is normally the $\beta$ subunit of the $\mathrm{Na}^{+} / \mathrm{K}^{+}$-ATPase. Indeed, our results show, that, as expected, ATP1B1 localizes at the basolateral membrane. However, under conditions of ATP12A upregulation (i.e., CF cells in vivo or cells treated with IL-4 in vitro), a significant fraction of ATP1B1 is moved to the apical membrane. It can be hypothesized that ATP12A competes with the $\mathrm{Na}^{+} / \mathrm{K}^{+}$-ATPase for the common $\beta$ subunit. In agreement with this hypothesis, we found that ATP1B1 is apically localized in the colon of normal mice but is displaced to 
A ATP12A+ATP1B1

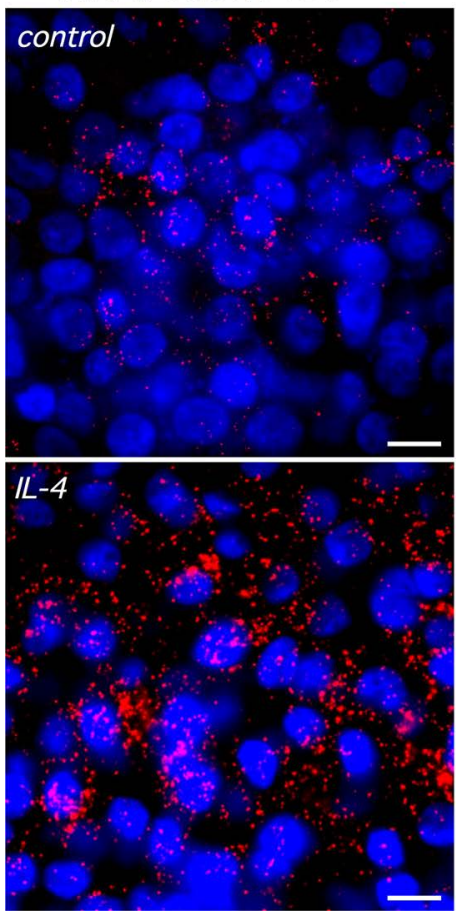

ATP12A+ATP4B

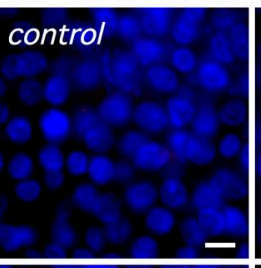

\section{IL-4}

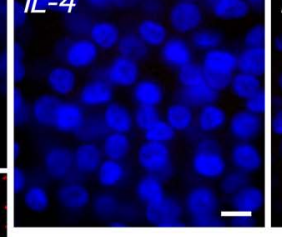

control
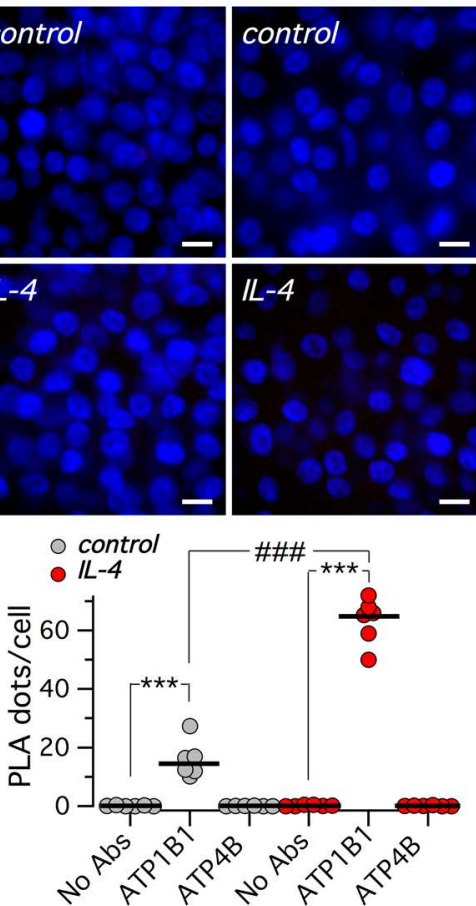

B
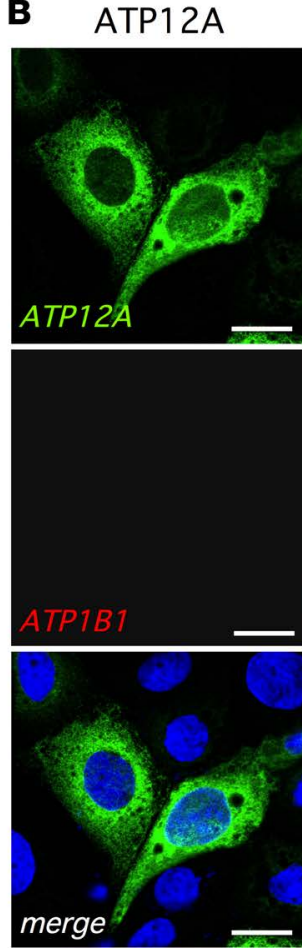

ATP12A+ATP1B1
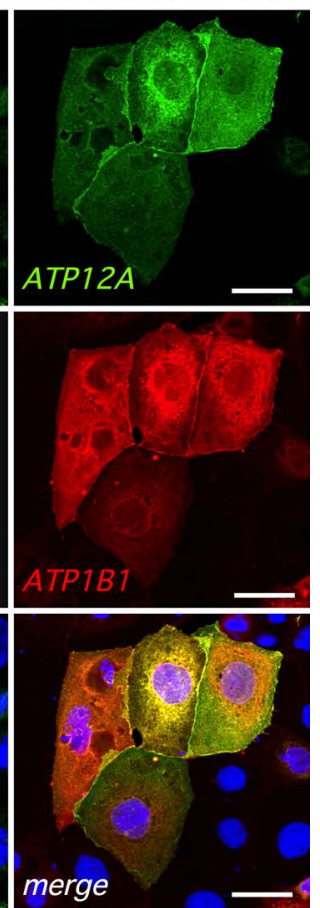

Figure 6. ATP12A-ATP1B1 proximity. (A) Representative images from proximity ligation assay (PLA) performed on cultured bronchial epithelial cells treated with and without IL-4 for 72 hours. Evidence of proximity (distance $<40 \mathrm{~nm}$ ) between ATP12A and ATP1B1 is indicated by the appearance of red dots. Nuclei are counterstained with DAPI (blue). Scale bar: $10 \mu \mathrm{m}$. No positive PLA reaction (i.e., red dots) was observed for ATP12A and ATP4B or in negative control (i.e., without primary antibodies, no Abs). The dot plot reports the quantification of PLA signal, measured as dots per cell; ${ }^{* * *} P<0.001$ vs. no Abs; ${ }^{\# \# P<0.001 ~ v s . ~ u n t r e a t e d ~(~} n=6$ for each condition). (B) Representative confocal images showing immunodetection of ATP12A and ATP1B1 in CFBE41o- cells transfected with ATP12A alone (left) or in combination with ATP1B1 (right). Scale bar: $10 \mu \mathrm{m}$.

lateral membranes in ATP12A-KO animals. Future experiments will need to conclusively assess if there is a direct competition between the respective $\alpha$ subunits of the 2 pumps for ATP1B1 and to identify the protein domains that are involved in these interactions.

We also measured $\mathrm{pH}$ of the apical fluid in vitro to estimate the activity of ATP12A. Compared with a previous study by our group (5), we have now considerably shortened the time of fluid application on the apical surface ( 3 hours instead of 48 hours). In addition, we have also included CF epithelia in our experiments. Our results raise several points for consideration. First, we detected a difference in $\mathrm{pH}$ between $\mathrm{CF}$ and non-CF epithelia under untreated (no IL-4) conditions. The lower $\mathrm{pH}$ in $\mathrm{CF}$ epithelia, in agreement with previous studies $(3,11,25,27)$, can be explained as the combined result of the loss of CFTR-dependent bicarbonate secretion and the proton pumping carried out by ATP12A. In agreement with this explanation, removal of bicarbonate acidified the apical fluid in non-CF epithelia. Importantly, apical ouabain minimized the difference in $\mathrm{pH}$ between $\mathrm{CF}$ and non-CF epithelia. Therefore, our findings demonstrate that ATP12A function is an important factor in the regulation of $\mathrm{pH}$ on the apical surface of airway epithelia. However, a recent study has shown no difference in $\mathrm{pH}$ between $\mathrm{CF}$ and non-CF in vivo and in vitro (9). The reason for these discrepant results is unclear. They may be due to different experimental methodologies but may also be attributed to the type of patients used for the study. Importantly, Schultz et al. have involved patients younger than 6 years of age (i.e., at an early stage of the disease; ref. 9). Instead, our study has been done on bronchial epithelial cells obtained from lung transplants (i.e., from adults and, in case of $\mathrm{CF}$ patients, from individuals at an advanced stage of the disease). It will be important to assess in future studies if $\mathrm{pH}$ regulation and ATP12A expression are affected by these factors.

The second point to be considered regards the effect of IL-4. As discussed above, in a previous study, we kept the apical fluid on the cells for 48 hours (5). After collection of the fluid, we found that the $\mathrm{pH}$ and the bicarbonate concentration were markedly increased by IL- 4 treatment. For the present study, we have applied the fluid for only 3 hours. Despite the shortened time, our results confirm our previous findings: 

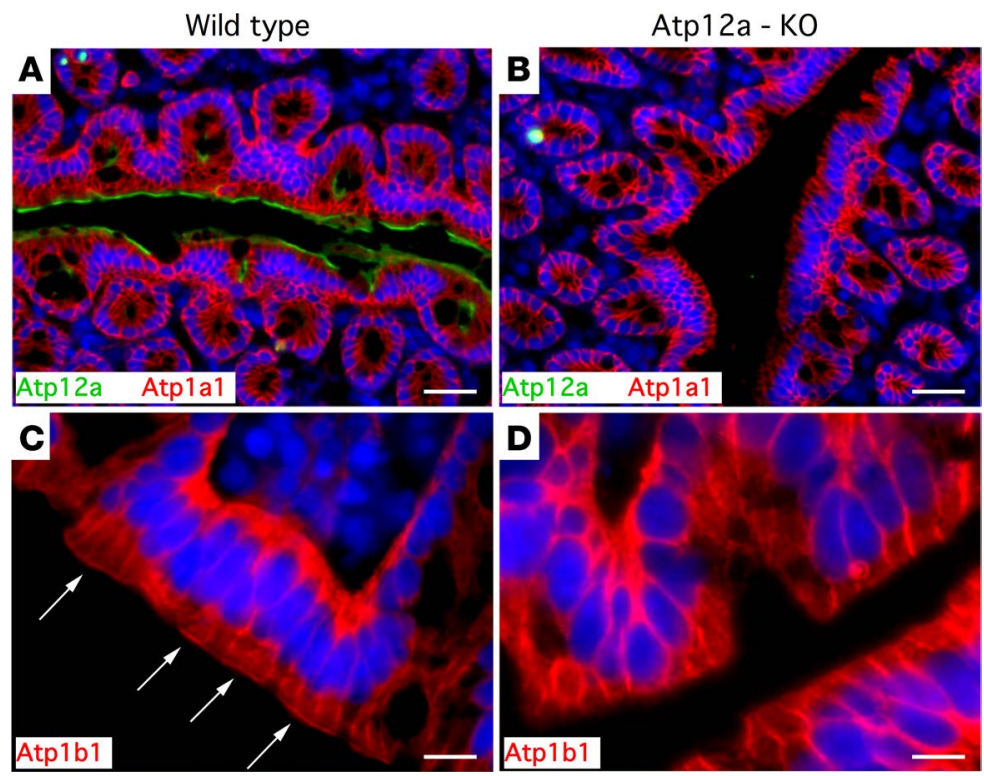

Figure 7. Expression of ATP12A and ATP1B1 in mice. Images show histological sections of murine colon with immunodetection of Atp12a and the $\alpha 1$ subunit of $\mathrm{Na}^{+} / \mathrm{K}^{+}$-ATPase (Atp1a1) (top) or Atp1b1 alone (bottom) in WT (A and C) or Atp12a-KO (B and D) animals. Arrows show expression of Atp1b1 in the apical membrane of WT animals. Scale bars: $20 \mu \mathrm{m}$ for panels $\mathbf{A}$ and $\mathbf{B} ; 10 \mu \mathrm{m}$ for panels $\mathbf{C}$ and $\mathbf{D}$. The images are representative of results obtained from 3 WT and 3 Atp12a-KO mice.

epithelia treated with IL-4 markedly increase the $\mathrm{pH}$ on the apical surface. This effect, which was also similarly observed in CF epithelia, is dependent on enhanced bicarbonate transport. Indeed, removal of bicarbonate in the basolateral solution resulted in marked acidification, an effect that was blocked by ouabain, an inhibitor of ATP12A. The similar $\mathrm{pH}$ values observed in CF and non-CF epithelia after IL-4 treatment (despite the difference observed in untreated conditions) suggest that the cytokine markedly enhances the contribution of CFTR-independent over CFTR-dependent bicarbonate transport mechanisms. This finding may have important implications. An upregulation of CFTR-independent mechanisms, induced by cytokines or other soluble mediators in vivo and possibly related to age and extent/type of inflammation, could increase ASL pH despite the concomitant upregulation of ATP12A. The complexity of such mechanisms can explain the conflicting results reported for ASL $\mathrm{pH}$. In this respect, an in vivo study found a difference between CF and non-CF infants (28). However, this difference was not present when older individuals were examined (28). It should be also noted that, in another study, treatment of cultured airway epithelial cells with IL-13 resulted in acidification and not alkalinization of apical fluid, as in our experiments (29). IL-4 and IL-13 are both Th2 cytokines that cause goblet cell hyperplasia with similar mechanisms. However, subtle differences in their effect on expression and function of channels and transporters (e.g., ATP12A, non-CFTR bicarbonate transporters) could explain the different effect on ASL $\mathrm{pH}$.

We obtained interesting results by using ouabain on epithelia treated with IL-4. In the absence of bicarbonate, ouabain produced the expected increase in $\mathrm{pH}$. However, with bicarbonate, ouabain was essentially ineffective. Such results could suggest that ATP12A, despite the increased expression, is not active in the presence of bicarbonate. In this respect, we also measured $\mathrm{K}^{+}$concentration in the apical fluid as another readout of ATP12A function. We found a marked depletion of apical $\mathrm{K}^{+}$in cells treated with IL-4 and kept in bicarbonate. These results indicate that ATP12A is indeed active under these conditions. In agreement with this interpretation, we found that $\mathrm{K}^{+}$absorption is sensitive to ouabain. There is an intriguing hypothesis to explain the lack of $\mathrm{pH}$ change when using ouabain on cells treated with IL- 4 and kept with bicarbonate. It is possible that, under these conditions, $\mathrm{K}^{+}$is, in part, exchanged with $\mathrm{Na}^{+}$and not with $\mathrm{H}^{+}$. Actually, previous in vitro studies have postulated that ATP12A may also work as an apical $\mathrm{Na}^{+} / \mathrm{K}^{+}$pump $(30,31)$, a result recently confirmed in vivo and contributing to renal salt excretion (32). We wondered whether the $\mathrm{K}^{+}$depletion observed in our experiments is consistent with the affinity of ATP12A for this cation. It has been reported that human and rat ATP12A have a relatively high affinity, with a $\mathrm{K}_{1 / 2}$ (i.e., the $\mathrm{K}^{+}$concentration required to have $50 \%$ of transporter activity) of $0.7-1.0 \mathrm{mM}(33,34)$. Therefore, the pump is expected to be active at low concentrations of extracellular $\mathrm{K}^{+}$, although at a reduced rate. 
A

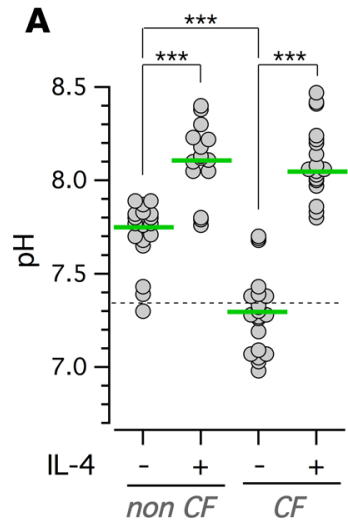

B

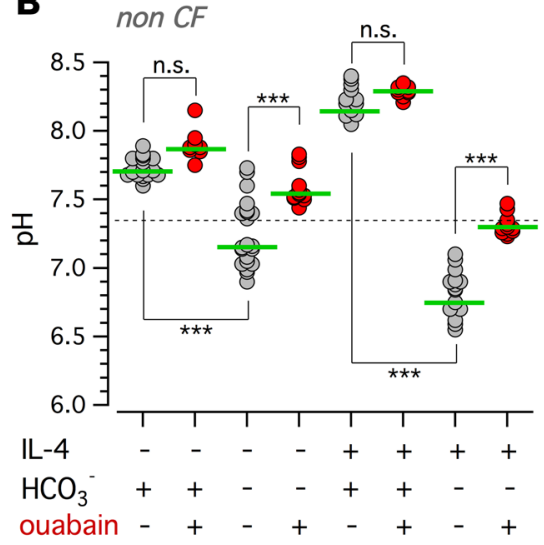

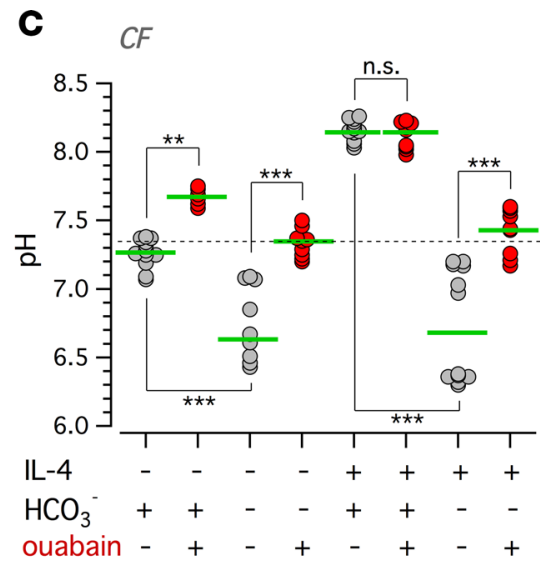

\section{D}

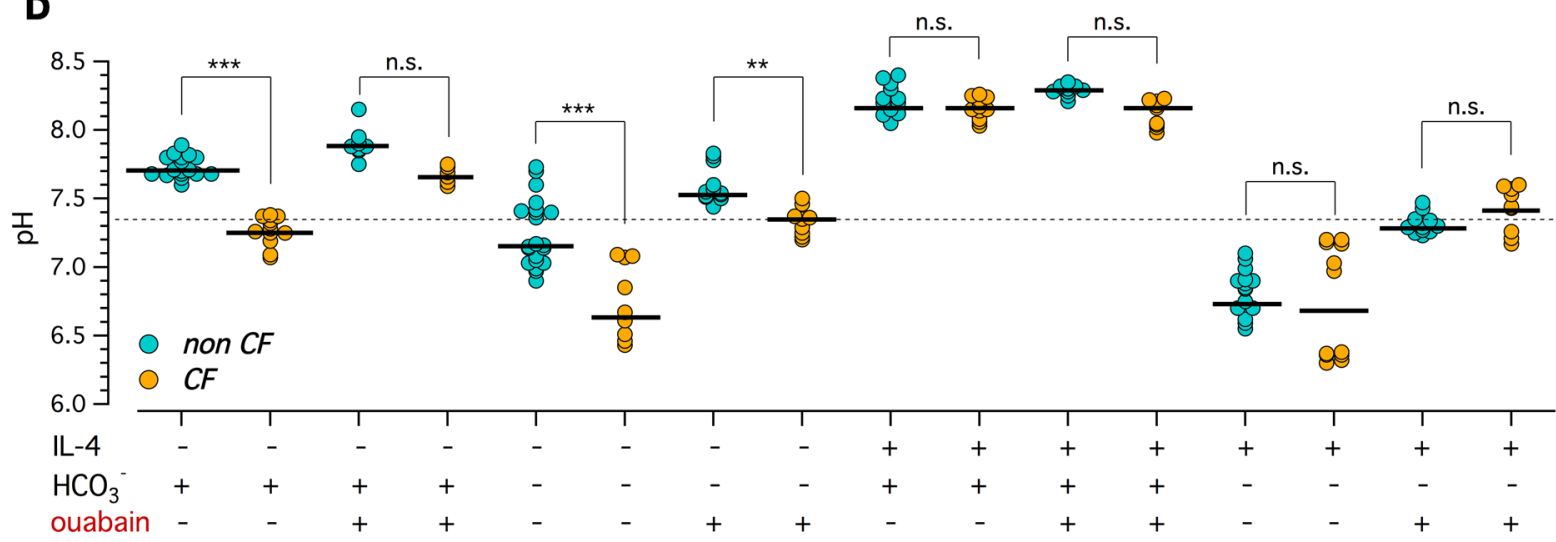

Figure 8. Apical fluid pH measurement. (A) Dot plot (single values and median) showing apical fluid pH measured in cultured CF and non-CF bronchial epithelia treated with/without IL-4 for 72 hours ( ${ }^{* *} P<0.001$; data collected from 6 CF and 6 non-CF bronchial cell preparations; $n=15-18$ separate epithelia for each condition). (B and C) Dot plots (single values and median) showing apical fluid pH measured in non-CF (B) and CF (C) bronchial epithelia treated with/without IL-4 for 72 hours. Before pH measurement, cells were incubated for 3 hours with a basolateral medium containing $\mathrm{HCO}_{3}{ }^{-}$or $\mathrm{HEPES}$ and with $75 \mu \mathrm{l}$ of a modified PBS solution on the apical side. Where indicated, this solution also contained $200 \mu \mathrm{M}$ ouabain $\left({ }^{* *} P<0.01,{ }^{* * *} P<0.001\right.$; data collected from 4 Îseparate CF and non-CF bronchial cell preparations). (D) The data reported in B and C are replotted to directly compare side-by-side CF and non-CF data for each condition. The dashed line in each panel indicates the initial $\mathrm{pH}$ of the saline solution.

In conclusion, our studies have produced important findings related to ATP12A. The upregulation in the airways of CF patients and, possibly, in other individuals with bacterial infections of the airways can be a pathogenic factor. It will be important in future studies to assess if ATP12A upregulation is an early feature of $\mathrm{CF}$ or is a characteristic that emerges at a more advanced stage of the disease. Another finding is the discovery of ATP1B1 as the partner required by ATP12A for trafficking to the plasma membrane. This finding also has important practical implications because it will allow generation of cell models expressing both proteins for functional and pharmacological studies. We also obtained results showing that ATP12A indeed contributes to the regulation of $\mathrm{pH}$ and/or $\mathrm{K}^{+}$concentration in the ASL. The net effect on $\mathrm{pH}$ may be affected by the concomitant activity of CFTR-independent bicarbonate transport systems. Regarding $\mathrm{K}^{+}$, it should be noted that this cation has an important role in the signaling within bacteria communities. Waves of potassium released by bacteria enable long-range electrical communication in bacterial biofilms (35). Therefore, the marked depletion of $\mathrm{ASL} \mathrm{K}^{+}$produced by ATP12A deserves particular attention to understand if it is a byproduct of proton pumping or a key function in the airway epithelium.

\section{Methods}

Immunofluorescence of human bronchial samples. Tissue samples were obtained from 22 patients undergoing lung transplant (15 CF and 7 non-CF). Samples were fixed in 10\% neutral buffered formalin (05-01005Q, Bio-Optica), embedded in paraffin and sectioned at $7 \mu \mathrm{m}$. For immunofluorescence detection of ATP12A protein, sections were deparaffinized and subjected to antigen retrieval with $10 \mathrm{mM}$ citrate buffer ( $\mathrm{pH}$ 6) heated to $95^{\circ} \mathrm{C}$ in a microwave for 5 minutes. Samples were then cooled to room temperature 

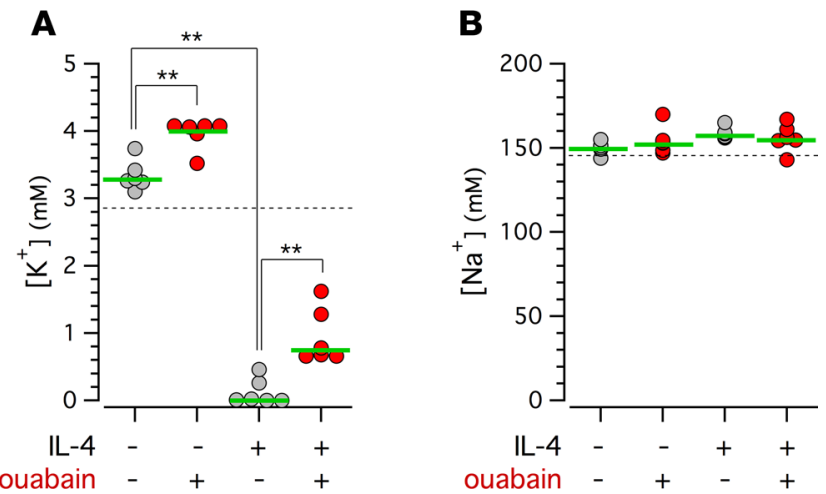

Figure 9. Ouabain-sensitive $\mathrm{K}^{+}$absorption. Dot plots (single values and median) report the $\mathrm{K}^{+}(\mathbf{A})$ and $\mathrm{Na}^{+}$(B) concentration in the apical fluid collected from epithelia treated with/without IL-4 for 72 hours (data collected from 2 separate preparations of non-CF cells). Where indicated, the fluid contained ouabain. The horizontal dashed lines indicate the original concentration of the cation in the fluid.

and permeabilized with Triton X-100 $0.3 \%$ in PBS for 5 minutes. After washing, cells were blocked with $1 \%$ BSA in PBS for 2 hours, and then incubated overnight at $4^{\circ} \mathrm{C}$ with primary antibodies diluted in PBS containing 1\% BSA. The following antibodies and dilutions were used: rabbit anti-ATP12A (HPA039526, MilliporeSigma) at 1:400; mouse IgG1 anti-MUC5AC (MA5-12178, Thermo Fisher Scientific) at 1:200; mouse IgG2B anti-acetylated tubulin (7451, MilliporeSigma) at 1:300; and mouse IgG2A anti-ATP1B1 (ab2873, Abcam) at 1:1,000.

Following incubation with primary antibodies, tissues were rinsed 3 times in PBS and incubated with a solution of secondary goat anti-rabbit Alexa Fluor 488, goat anti-mouse IgG1 Alexa Fluor 546, and goat anti-mouse IgG2B Alexa Fluor 633 antibodies (Thermo Fisher Scientific) diluted at 1:200 in PBS containing 1\% BSA for 1 hour in the dark. After further 3 washes in PBS, slides were mounted with Fluoroshield with DAPI (MilliporeSigma) to stain cell nuclei.

Confocal microscopy was performed using a laser scanning confocal microscope TCS SPE (Leica Microsystems). Image analysis was performed using Leica and ImageJ (NIH) software.

Cell culture. The procedures for isolation and culture of human bronchial epithelial cells were described in detail in a previous study (16). Briefly, mainstem human bronchi, derived from $\mathrm{CF}$ and non-CF individuals undergoing lung transplant, were dissected, washed, and incubated overnight at $4^{\circ} \mathrm{C}$ in protease XIV solution. Epithelial cells were then detached mechanically, dissociated by trypsinization, and cultured in flasks in a serum-free medium (LHC basal medium/RPMI 1640, Thermo Fisher Scientific) supplemented with various hormones and supplements; hormones and supplements as specified in ref. 16. With respect to the previous study, this culture medium also contained BMP antagonist (DMH-1; Tocris), TGF $\beta$ antagonist (A 83-01; Tocris), and ROCK1 inhibitor (Y-27632; Tocris), a cocktail of compounds that allows expansion of basal stem cells (36). After 4-5 passages, cells were seeded at high density $\left(500,000 / \mathrm{cm}^{2}\right)$ on Snapwell 3801 (Corning Costar) porous inserts. After 24 hours from seeding, the medium was switched to DMEM/ F12 (1:1) plus 2\% New Zealand FBS (Thermo Fisher Scientific), hormones, and supplements (16) to reprogram the cells and induce mucociliary differentiation. The medium was replaced daily on both sides of permeable supports up to 7 days (liquid-liquid culture; LLC). Subsequently, the apical medium was totally removed and the cells received nutrients only from the basolateral side (air-liquid culture; ALC). This condition favored a further differentiation of the epithelium. Cells were maintained under ALC for 2 weeks.

Cells were obtained from 3 non-CF subjects (HBE9, HBE10, HBE11) and 3 CF subjects (CFBE17, CFBE18, CFBE19: patients with F508del/F508del genotype). For CF cells, the LHC basal medium/ RPMI 1640 medium also contained, in the first 4 days, additional antibiotics to eradicate bacterial contamination. For this purpose, the mixture of antibiotics (usually colistin, piperacillin, and tazobactam) and dosage were designed on the basis of the antibiogram of bacteria isolated from the most recent expectorate of the patient.

Where indicated, well-differentiated bronchial epithelial cells were treated with or without IL-4 (10 ng/ $\mathrm{ml}$ in the basolateral medium; MilliporeSigma) or bacterial supernatant (diluted 1:8 into PBS; $200 \mu 1$ added to the apical side) for 72 hours (37). 
Preparation of bacterial supernatants. P. aeruginosa (PA), strain ATCC27853, was used for the preparation. Bacteria were grown in LB broth (Difco) at $37^{\circ} \mathrm{C}$ with shaking at $250 \mathrm{rpm}$ for 24 hours (to $\mathrm{OD}_{600} \sim 5$ ). Bacterial cultures were centrifuged at 5,000 $\mathrm{g}$ for 10 minutes, and the supernatants were sterile filtered with low-protein binding $0.22 \mu \mathrm{m}$ cellulose acetate filters (Corning) and stored at $-80^{\circ} \mathrm{C}$ until use.

Immunofluorescence of cultured bronchial epithelial cells. Primary human bronchial epithelial cells differentiated on Snapwell permeable supports were fixed by adding $200 \mu 1$ of $10 \%$ neutral buffered formalin (0501005Q, Bio-Optica) to the apical side for 5 minutes at room temperature. After 3 washings in PBS, cells were processed for antigen retrieval with $10 \mathrm{mM}$ citrate buffer $\left(\mathrm{pH}\right.$ 6) heated to $95^{\circ} \mathrm{C}$ in a microwave for 5 minutes. Samples were then cooled to room temperature, permeabilized with Triton X-100 0.3\% in PBS for 5 minutes, blocked with $1 \%$ BSA in PBS for 2 hours, and then incubated overnight at $4^{\circ} \mathrm{C}$ with $200 \mu 1$ of primary antibodies diluted in PBS containing 1\% BSA. The following primary antibodies and dilutions were used: rabbit anti-ATP12A (HPA039526, MilliporeSigma) at 1:400; mouse IgG1 anti-MUC5AC (MA512178, Thermo Fisher Scientific) at 1:200; mouse IgG2B anti-acetylated tubulin (7451, MilliporeSigma) at 1:300; mouse anti-ATP4B (ab2866, Abcam) at 1:1000; mouse IgG2A anti-ATP1B1 (ab2873, Abcam) at 1:1,000; rabbit anti-ATP1B1 (SpETb1) and rabbit anti-ATP1B2 (SpETb2) at 1:1,000 (antibodies generated and used as described in ref. 19); rabbit anti-ATP1B3 (ab137055, Abcam) at 1:100; mouse anti-ATP1A1 (05-369, Millipore) at 1:1,000.

Following incubation with primary antibody, cells were rinsed 3 times in PBS and incubated with 200 $\mu 1$ of a solution of secondary Alexa Fluor-conjugated antibodies (Invitrogen) diluted 1:200 in PBS containing 1\% BSA for 1 hour in the dark. After further 3 washes in PBS, the porous membrane carrying the cells was cut from the plastic support of the Snapwell, placed on microscope slides, and mounted with Fluoroshield with DAPI (MilliporeSigma) to stain cell nuclei.

Image acquisition was performed using a laser scanning confocal microscope TCS SPE (Leica Microsystems) and a LSM 880 Elyra Superresolution microscope (Zeiss). Image analysis was performed using Leica, Zeiss, and ImageJ software.

Western blot. Cells were lysed in RIPA $1 \times$ buffer $(50 \mathrm{mM}$ Tris- $\mathrm{HCl} \mathrm{pH}$ 7.4, $150 \mathrm{mM} \mathrm{NaCl}, 1 \%$ Triton $\mathrm{X}-100,0.5 \%$ sodium deoxycholate, $0.1 \%$ SDS) containing Complete Protease Inhibitor Cocktail (Roche Diagnostics). Protein concentration in lysates was quantified using the DC Protein Assay kit (Bio-Rad). Lysates (20 $\mu$ g of total) were separated onto Criterion TGX precast Gels 4-15\% (Bio-Rad) and transferred to PVDF membrane (Bio-Rad) for Western blotting with Trans-Blot Turbo system (BioRad). ATP12A protein was immunodetected by rabbit anti-ATP12A (HPA039526, MilliporeSigma) at 1:4,000, followed by anti-rabbit HRP (Dako) 1:50,000 secondary antibody.

Membranes were also stripped with the Restore Western Blot Stripping buffer (Thermo Fisher Scientific) and incubated with the mouse monoclonal anti-GAPDH antibody clone 6C5 (Santa Cruz Biotechnology Inc.) 1:10,000, followed by anti-mouse HRP-conjugated secondary antibody (Dako; 1:50,000). All antibodies were dissolved in 5\% skimmed milk in TBS-T. Protein bands were visualized using the Super Signal West Femto Substrate (Thermo Fisher Scientific). Direct recording of the chemiluminescence was performed using the Alliance MINI HD9 AUTO Western Blot Imaging System (Biocompare).

Proximity ligation assay. Cultured bronchial cells treated with or without IL-4 (10 ng/ml) were fixed, subjected to antigen retrieval, and permeabilized as described for immunofluorescence experiments. ATP12AATP1B1 interaction, revealed as red fluorescent dots, was detected using the Duolink PLA kit (DUO92008, DUO82049, DUO92004, DUO92002; MilliporeSigma), according to the manufacturer's instructions. The following couples of primary antibodies were used: rabbit anti-ATP12A (HPA039526, MilliporeSigma), mouse anti-ATP1B1 (ab2873, Abcam), rabbit anti-ATP12A (HPA039526, MilliporeSigma), and mouse antiATP4B (ab2866, Abcam). After incubation with PLA probes, ligation, and amplification steps, the porous membrane carrying the cells was cut from the plastic support of the Snapwell, placed on microscope slides, and mounted with Fluoroshield with DAPI (MilliporeSigma) to stain cell nuclei.

Image acquisition was performed using an Olympus fluorescence microscope equipped with Lamda DG4 illuminator (Sutter Instruments) and Prime sCMOS camera (Photometrics). PLA dot quantification was performed with ImageJ software.

Transfection. CFBE41o- cells were cultured in minimal essential medium (MEM; Thermo Fisher Scientific) supplemented with 10\% FCS (MilliporeSigma), $2 \mathrm{mM} \mathrm{L-glutamine,} 100 \mathrm{U} / \mathrm{ml}$ penicillin, and 100 $\mu \mathrm{g} / \mathrm{ml}$ streptomycin. For immunofluorescence detection of ATP12A protein trafficking to the plasma membrane, CFBE41o- cells were seeded in a $\mu$-Chamber 12 well (Ibidi) at a density of 25,000 cells/well. 
After 24 hours, cells were transfected with plasmids carrying the coding sequence for human ATP12A and ATP1B1. For each well, $0.2 \mu \mathrm{g}$ of plasmid DNA and $0.5 \mu$ of Lipofectamine 2000 (Thermo Fisher Scientific) were first premixed in $50 \mu 1$ of Opti-MEM (Thermo Fisher Scientific) to generate transfection complexes (60 minutes at room temperature) and were then added to the cells. After 24 hours, the complexes were removed by replacing with fresh culture medium. After a further 24 hours, cells were fixed and processed for immunofluorescence experiments as described for primary cultures.

Image acquisition and analysis were performed using a laser scanning confocal microscope TCS SPE (Leica Microsystems) and ImageJ software, respectively.

Apical fluid $\mathrm{pH}$ and $\mathrm{K}^{+}$measurements. Differentiated bronchial epithelia under ALC conditions were treated for 72 hours with or without IL-4 $(10 \mathrm{ng} / \mathrm{ml})$. At the end of the treatment, cells were incubated $\left(37^{\circ} \mathrm{C}\right.$, $5 \% \mathrm{CO}_{2}$ atmosphere) with bicarbonate- or HEPES ( $25 \mathrm{mM}$ )-buffered medium on the basolateral side and $75 \mu \mathrm{l}$ of a modified PBS solution with low buffer capacity on the apical side. The modified PBS solution had the following composition: $145 \mathrm{mM} \mathrm{NaCl}, 2.7 \mathrm{mM} \mathrm{KCl}, 0.81 \mathrm{mM} \mathrm{Na} \mathrm{HPO}_{4}, 0.15 \mathrm{mM} \mathrm{KH}_{2} \mathrm{PO}_{4}$, $1 \mathrm{mM} \mathrm{CaCl}_{2}, 0.5 \mathrm{mM} \mathrm{MgCl}_{2}, \mathrm{pH} 7.35$. Where indicated, the modified PBS solution also contained ouabain (200 $\mu \mathrm{M}$, O3125; MilliporeSigma). After 3 hours, the apical fluid was recovered in a single step (i.e., without repeated pipetting), and $\mathrm{pH}$ was measured with a microelettrode (PHR-146S, Lazar Research Laboratories) under ambient air. Similarly, after recovery, the $\mathrm{K}^{+}$concentration of the apical fluid was determined by flame photometry (IL943, Instruments Laboratory).

Mouse models. Experiments were performed on C57B16 WT and KO mice for the HKA2 $\alpha$ (ATP12A) subunit gene (38). All the animals were kept at CEF (Centre d'Explorations Fonctionnelles of CRC, Agreement no. A75-06-12). Anesthetized mice (10 mg/kg xylazine and $100 \mathrm{mg} / \mathrm{kg}$ ketamine) were perfused with $4 \%$ paraformaldehyde in the aorta, and the colons were removed and frozen in OCT. Slices ( $5 \mu \mathrm{m}$ thick) were then processed for immunofluorescence microscopy using a home-made polyclonal anti-ATP12A antibody (1:200) and/or a monoclonal anti-ATP1A1 ( $\alpha 1$ subunit of the Na,K-ATPase, 1:1,000) and/or the anti-ATP1B1 (SpETb1, 1:1,000).

Statistics. Data are presented as representative images, as dot plots with medians, or as graphs with mean \pm SEM. Statistical significance of differences among groups of data was verified by 1-way ANOVA followed by the Tukey post-hoc test. For data not passing the normality test, a nonparametric ANOVA was used. A $P$ value lower than 0.05 was considered significant.

Study approval. All procedures related to the use of human epithelial cells were carried out in accordance with approved guidelines. In particular, the protocols to isolate, culture, store, and study bronchial epithelial cells from patients undergoing lung transplant was approved by the local ethical committee under the supervision of the Italian Ministry of Health. Written informed consent was obtained from all patients using a form that was also approved by the same ethical committee. All experiments were conducted in accordance with the institutional guidelines and the recommendations for the care and use of laboratory animals put forward by the Directive 2010/63/EU on the protection of animals used for scientific purposes.

\section{Author contributions}

PS, IM, EC, AV, and PM did the experiments on cultured cells; PS, AV, DT, AP, and PMV worked on the analysis of protein expression in bronchial samples; GC evaluated protein expression in mice; PS, CW, GP, and GC measured $\mathrm{pH}$ and ion composition in apical fluid; PS, ISG, GP, GC, and LJVG analyzed the data and prepared the manuscript.

\section{Acknowledgments}

This study was supported by grants from the Cystic Fibrosis Foundation (GALIET17G0) and from Telethon Foundation (TMLGCBX16TT) to LJVG and by recurrent grants from the Institut National de la Santé et de la Recherche Médicale (INSERM) and from the Centre National de la Recherche Scientifique (CNRS). We are grateful for the technical assistance of the "platforme d'exploration fonctionnelle du petit animal" of the team "Métabolisme et Physiologie Rénale" at the Centre de Recherche des Cordeliers.

Address correspondence to: Luis J.V. Galietta, Telethon Institute of Genetics and Medicine, Via Campi Flegrei 34, 80078 Pozzuoli NA, Italy. Phone: 39.081.1923.0693; Email: 1.galietta@tigem.it. 
1. Haq IJ, Gray MA, Garnett JP, Ward C, Brodlie M. Airway surface liquid homeostasis in cystic fibrosis: pathophysiology and therapeutic targets. Thorax. 2016;71(3):284-287.

2. Smith JJ, Welsh MJ. cAMP stimulates bicarbonate secretion across normal, but not cystic fibrosis airway epithelia. J Clin Invest. 1992;89(4):1148-1153.

3. Shah VS, et al. Airway acidification initiates host defense abnormalities in cystic fibrosis mice. Science. 2016;351(6272):503-507

4. Garnett JP, et al. Novel role for pendrin in orchestrating bicarbonate secretion in cystic fibrosis transmembrane conductance regulator (CFTR)-expressing airway serous cells. J Biol Chem. 2011;286(47):41069-41082.

5. Gorrieri G, et al. Goblet Cell Hyperplasia Requires High Bicarbonate Transport To Support Mucin Release. Sci Rep. 2016;6:36016

6. Pezzulo AA, et al. Reduced airway surface $\mathrm{pH}$ impairs bacterial killing in the porcine cystic fibrosis lung. Nature. 2012;487(7405):109-113.

7. Hoegger MJ, et al. Impaired mucus detachment disrupts mucociliary transport in a piglet model of cystic fibrosis. Science. 2014;345(6198):818-822.

8. Tang XX, et al. Acidic pH increases airway surface liquid viscosity in cystic fibrosis. J Clin Invest. 2016;126(3):879-891.

9. Schultz A, et al. Airway surface liquid $\mathrm{pH}$ is not acidic in children with cystic fibrosis. Nat Commun. 2017;8(1):1409.

10. Crambert G. H-K-ATPase type 2: relevance for renal physiology and beyond. Am J Physiol Renal Physiol. 2014;306(7):F693-F700.

11. Coakley RD, et al. Abnormal surface liquid $\mathrm{pH}$ regulation by cultured cystic fibrosis bronchial epithelium. Proc Natl Acad Sci USA. 2003;100(26):16083-16088

12. Min JY, et al. Proton pump inhibitors decrease eotaxin-3/CCL26 expression in patients with chronic rhinosinusitis with nasal polyps: Possible role of the nongastric H,K-ATPase. J Allergy Clin Immunol. 2017;139(1):130-141.e11.

13. Lennox AT, et al. ATP12A promotes mucus dysfunction during Type 2 airway inflammation. Sci Rep. 2018;8(1):2109.

14. Kim CH, Kim KE, Yoon JH, Song KS. Upregulation of MUC5AC gene expression by IL-4 through CREB in human airway epithelial cells. J Cell Biochem. 2009;108(4):974-981.

15. Lachowicz-Scroggins ME, Boushey HA, Finkbeiner WE, Widdicombe JH. Interleukin-13-induced mucous metaplasia increases susceptibility of human airway epithelium to rhinovirus infection. Am J Respir Cell Mol Biol. 2010;43(6):652-661.

16. Scudieri P, et al. Association of TMEM16A chloride channel overexpression with airway goblet cell metaplasia. J Physiol (Lond). 2012;590(23):6141-6155.

17. Garcia MA, Yang N, Quinton PM. Normal mouse intestinal mucus release requires cystic fibrosis transmembrane regulatordependent bicarbonate secretion. J Clin Invest. 2009;119(9):2613-2622.

18. Gustafsson JK, et al. Bicarbonate and functional CFTR channel are required for proper mucin secretion and link cystic fibrosis with its mucus phenotype. J Exp Med. 2012;209(7):1263-1272.

19. González-Martínez LM, Avila J, Martí E, Lecuona E, Martín-Vasallo P. Expression of the beta-subunit isoforms of the Na,K-ATPase in rat embryo tissues, inner ear and choroid plexus. Biol Cell. 1994;81(3):215-222.

20. Mobasheri A, et al. $\mathrm{Na}+, \mathrm{K}+$-ATPase isozyme diversity; comparative biochemistry and physiological implications of novel functional interactions. Biosci Rep. 2000;20(2):51-91.

21. Adams G, Tillekeratne M, Yu C, Pestov NB, Modyanov NN. Catalytic function of nongastric H,K-ATPase expressed in Sf-21 insect cells. Biochemistry. 2001;40(19):5765-5776.

22. Reinhardt J, Grishin AV, Oberleithner H, Caplan MJ. Differential localization of human nongastric $\mathrm{H}(+)-\mathrm{K}(+)$-ATPase ATP1AL1 in polarized renal epithelial cells. Am J Physiol Renal Physiol. 2000;279(3):F417-F425.

23. Pestov NB, Korneenko TV, Radkov R, Zhao H, Shakhparonov MI, Modyanov NN. Identification of the beta-subunit for nongastric H-K-ATPase in rat anterior prostate. Am J Physiol, Cell Physiol. 2004;286(6):C1229-C1237.

24. Pestov NB, Korneenko TV, Shakhparonov MI, Shull GE, Modyanov NN. Loss of acidification of anterior prostate fluids in Atp12a-null mutant mice indicates that nongastric H-K-ATPase functions as proton pump in vivo. Am J Physiol, Cell Physiol. 2006;291(2):C366-C374.

25. Garland AL, et al. Molecular basis for $\mathrm{pH}-$ dependent mucosal dehydration in cystic fibrosis airways. Proc Natl Acad Sci USA 2013;110(40):15973-15978

26. Smith JJ, Welsh MJ. Fluid and electrolyte transport by cultured human airway epithelia. J Clin Invest. 1993;91(4):1590-1597.

27. Shah VS, et al. Relationships among CFTR expression, HCO3- secretion, and host defense may inform gene- and cell-based cystic fibrosis therapies. Proc Natl Acad Sci USA. 2016;113(19):5382-5387.

28. Abou Alaiwa MH, et al. Neonates with cystic fibrosis have a reduced nasal liquid pH; a small pilot study. JCyst Fibros. 2014;13(4):373-377

29. Haggie PM, Phuan PW, Tan JA, Zlock L, Finkbeiner WE, Verkman AS. Inhibitors of pendrin anion exchange identified in a small molecule screen increase airway surface liquid volume in cystic fibrosis. FASEB J. 2016;30(6):2187-2197.

30. Cougnon M, Bouyer P, Planelles G, Jaisser F. Does the colonic H,K-ATPase also act as an Na,K-ATPase? Proc Natl Acad Sci USA 1998;95(11):6516-6520.

31. Grishin AV, Caplan MJ. ATP1AL1, a member of the non-gastric H,K-ATPase family, functions as a sodium pump. J Biol Chem. 1998;273(43):27772-27778.

32. Morla L, Doucet A, Lamouroux C, Crambert G, Edwards A. The renal cortical collecting duct: a secreting epithelium? J Physiol (Lond). 2016;594(20):5991-6008.

33. Cougnon M, Planelles G, Crowson MS, Shull GE, Rossier BC, Jaisser F. The rat distal colon P-ATPase alpha subunit encodes a ouabain-sensitive H+, K+-ATPase. J Biol Chem. 1996;271(13):7277-7280.

34. Crambert G, Horisberger JD, Modyanov NN, Geering K. Human nongastric H+-K+-ATPase: transport properties of ATP1al1 assembled with different beta-subunits. Am J Physiol, Cell Physiol. 2002;283(1):C305-C314.

35. Prindle A, Liu J, Asally M, Ly S, Garcia-Ojalvo J, Süel GM. Ion channels enable electrical communication in bacterial communities. Nature. 2015;527(7576):59-63.

36. Mou H, et al. Dual SMAD Signaling Inhibition Enables Long-Term Expansion of Diverse Epithelial Basal Cells. Cell Stem Cell. 2016;19(2):217-231.

37. Caci E, et al. Upregulation of TMEM16A Protein in Bronchial Epithelial Cells by Bacterial Pyocyanin. PLoS ONE 2015;10(6):e0131775.

38. Meneton $\mathrm{P}$, et al. Increased sensitivity to K+ deprivation in colonic H,K-ATPase-deficient mice. J Clin Invest. 1998;101(3):536-542. 